\title{
Reduction of energy losses achieved by an optimal reactive power generation in photovoltaic systems installed inside a low voltage distribution network
}

\author{
Ernest Belič, Katarina Dežan, Niko Lukač and Gorazd Štumberger \\ University of Maribor \\ Faculty of Electrical Engineering and Computer Science \\ Smetanova ulica 17, 2000 Maribor, Slovenia \\ Phone: +386 22207175 , Fax: +386 22207272 , \\ e-mail: ernest.belic@um.si, katarina.dezan@um.si, niko.lukac@um.si, gorazd.stumberger@um.si
}

\begin{abstract}
.
This paper deals with the evaluation of electrical energy savings in a low voltage distribution network, gathered by reduced energy losses. The latter is achieved by optimal reactive power generation of photovoltaic (PV) systems, where losses in the low voltage network and distribution transformer are taken into consideration. The energy savings are determined for three typical low voltage networks: urban, rural and rural with long distance LV lines. In the discussed networks PV systems are installed. The study of network electrical energy savings is performed for smaller PV systems with $5 \mathrm{kWp}$ per unit and for larger units, all for the same total PV power installed in the network. The results shows, that significant electrical energy savings can be achieved in networks, where PV systems are installed away from the transformer station or smaller PV systems are installed at sites, where consumption is present.
\end{abstract}

\section{Key words}

low voltage network, PV systems, energy loss reduction, energy savings

\section{Introduction}

The energy losses in low voltage distribution networks represent as much as $25 \%$ of the energy losses related with energy transmission, from big power plants through high and middle voltage network to the consumers in low voltage networks [1]. Another $25 \%$ of the total energy losses occur in the distribution transformers between middle to low voltage networks [1]. In order to reduce these losses, a proper capacitor placement, network reconfiguration and a proper placement of distributed generation units are normally applied [2]. In the low voltage networks, the capacitor placement is not a common approach. If it is done, then capacitors are installed in the transformer stations. In this way only the distribution transformer losses can be reduced. However, the losses in the low voltage network remain unchanged. The network reconfiguration seems to be a very efficient approach, at least to some extent. On the other hand, it requires large investments in additional switching devices and control technologies to achieve the aforementioned reduction of losses. Thus, the most promising measure for reduction of losses in low voltage networks seems to be the installation of properly sized and placed distributed generation units. If there are photovoltaic distributed generation units installed in the distribution network, they normally supply all produced energy to the network. That is why the installation of such units must be planned carefully to benefit in reduction of losses. If the large distributed generation units are installed directly to the transformer station, they can influence the losses in the distribution transformer. However, they cannot influence the losses in low voltage network. At this point it must be pointed out, that distributed generation units connected to the electricity network through an inverter, can supply also reactive power to the network. This can be done through several local control methods [3], considering mostly the voltage level at the point of common coupling, where losses in the distribution network are not included in the control algorithm. In order to use the reactive power generation also for reduction of losses, coordination between all distributed generation units in the network is required.

This paper focuses on the analysis of energy savings that can be achieved through a proper reactive power generation in distributed generation photovoltaic units connected to the low voltage network. The energy savings are achieved by the reduction of energy losses.

In the analysis performed, typical low voltage networks with distributed generators are observed. Due to the fact that currently larger distributed generation units are present in low voltage networks, the analysis is also done under assumption that small rooftop photovoltaic distributed generation units with the peak power under 5 $\mathrm{kWp}$ are installed in the discussed networks. The paper shows and evaluates the results of performed analysis.

\section{Evaluation of energy losses}

The energy losses are most frequently determined on the basis of previously performed load flow calculations. The load flow calculations serve as a tool to calculate all the node voltages and their angles, enabling evaluation of the energy flows in the entire network. Since the majority of 
low voltage networks operate in the radial configuration, backward - forward sweep method, similar to the one presented in [4], is used in this work. Based on the results of load flow calculation, the low voltage network losses can be determined using (1),

$$
P_{\text {loss }, L V}=\sum_{n=1}^{N_{b}}\left|\underline{I}_{n}\right|^{2} R_{n}
$$

where $P_{\text {loss, } L V}$ represents the energy losses in the observed low voltage network, $\underline{I}_{n}$ represents the current phasor in the branch $n, R_{n}$ represents the resistance of the branch $n$ and $N_{b}$ denotes the number of branches in the observed low voltage network. In the low voltage network losses calculated by (1), the losses due to the active and reactive power flows in the branch $n$ are considered through the current phasor $\underline{I}_{n}$. To take into account also losses in the distribution transformer, it is necessary to split the transformer losses into two parts [5]. These two parts are the core losses and the copper losses. The core losses $P_{F e}$, are subject to (2),

$$
P_{F e}=P_{F e 0} \frac{|\underline{U}|}{U_{N}}
$$

and the copper losses $P_{C u}$, are subject to (3).

$$
P_{C u}=P_{C u 0} \frac{|\underline{S}|^{2}}{S_{N}^{2}}
$$

In (2) and (3) $U_{N}$ and $S_{N}$ represent the transformer's rated voltage and power, $\underline{U}$ and $\underline{S}$ are the actual transformer voltage and transmitted power phasors, $P_{F e 0}$ are the core losses at the rated voltage and $P_{C u 0}$ are the copper losses at the rated power. Since the voltage at the transformer terminals in the normal operational states does not change substantially, the core losses are included in the load flow calculations as a constant power load. The transformer copper losses, on the other hand, vary with changing power flow through the transformer. Thus, the copper losses are included in load flow calculations through the resistive part of short circuit impedance. The short circuit impedance $Z_{T}$ is determined considering the short-circuit voltage $u_{k}$ in $\%$ (4)

$$
Z_{T}=\frac{u_{k}}{100} \frac{U_{N}^{2}}{S_{N}}
$$

and its resistive part $R_{T}$ as presented in (5).

$$
R_{T}=\frac{P_{C u 0}}{3 I_{N}^{2}}
$$

$I_{N}$ in (5) represents the transformer rated current. Finally, the transformer reaktance $X_{T}$ is calculated using (6).

$$
X_{T}=\sqrt{Z_{T}^{2}-R_{T}^{2}}
$$

The circuit model of the so obtained transformer model is presented in Fig. 1.

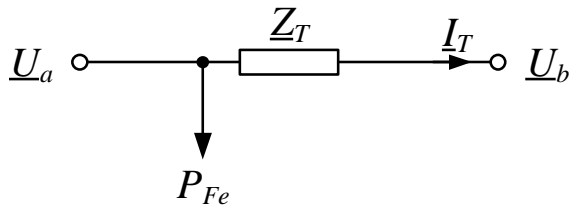

Fig. 1. Circuit model of the distribution transformer used in load flow calculations
$U_{a}$ and $\underline{U}_{b}$ represent the voltage phasors at both terminals of the transformer. Such circuit model of the distribution transformer does not take into account phase shifting caused by operation of the transformer. This is acceptable for this work, where only the low voltage network and distribution transformer losses are important.

After the results of load flow calculation are obtained, the copper losses are calculated by (7),

$$
P_{C u}=R_{T}\left|\underline{I}_{T}\right|^{2}
$$

whilst the total transformer losses $P_{\text {loss } T}$ are determined by (8).

$$
P_{\text {loss }, T}=P_{F e}+P_{C u}
$$

\section{Optimal reactive power generation in $\mathbf{P V}$ systems}

In general PV system can provide inductive or capacitive reactive power in accordance with their apparent power limits [6], described by (9).

$$
\left|Q_{G}\right|=\sqrt{S_{G, N}^{2}-P_{G}^{2}}
$$

The reactive power that can be generated in individual $\mathrm{PV}$ system depends on its rated apparent power $S_{G, N}$ and currently generated active power $P_{G}$. In general $\mathrm{PV}$ systems can generate reactive power that is lower that the generated active power. Thus, in this work it is considered that PV systems can generate reactive power up to the power factor of 0.8 .

In order to find an optimal distribution of reactive power generation among all PV systems present in the given low voltage distribution network, the objective function (10) must be minimized.

$$
q=\min \left(P_{\text {loss }, L V}+P_{\text {loss }, T}\right)
$$

In the minimization procedure, which is based on the load flow calculations, the following constrains must be fulfilled:

1) The reactive power generation of each PV system must met the limits (9) and its power factor must not be lower than 0.8 .

2) The voltage profile of the network must remain between limits specified in [7].

3) None of the elements in the observed distribution network (distribution transformer, low voltage lines) must be overloaded.

With minimization of the objective function (10) the optimal distribution of reactive power generation among individual PV systems can be determined for the given operating conditions. To evaluate the energy savings in time interval $m\left[t_{\mathrm{m}}, t_{\mathrm{m}+1}\right]$, in which it is assumed that conditions in the network have not changed, the difference between energy losses before (marked with old) and after optimization (marked with new) are determined (11).

$$
\Delta W_{\text {loss }, m}=\int_{t_{m}}^{t_{m+1}}\left(P_{\text {loss }, m}^{\text {old }}-P_{\text {loss }, m}^{\text {new }}\right) d t
$$

After that the energy savings in one year can be determined using (12) 


$$
W_{\text {loss }}=\sum_{m=1}^{N_{t}} \Delta W_{\text {loss }, m}
$$

where $N_{t}$ indicates number of time intervals in one year.

\section{Results}

In this section, 3 different low voltage networks are analysed regarding reduction of energy losses that can be achieved through the reactive power generation in PV systems. The network discussed can be described as: urban, rural and rural with long distance LV lines. The energy savings are evaluated for one year, where active powers of PV systems are determined on the basis of measured solar irradiance, represented as 12 characteristic days of the year, one for each month. Thus, the measured data is averaged for each 30 minute interval over each month in order to obtain the time dependent characteristic for 12 days representing months. The obtained time dependent characteristics of solar irradiance $J$ are presented in Fig. 2.

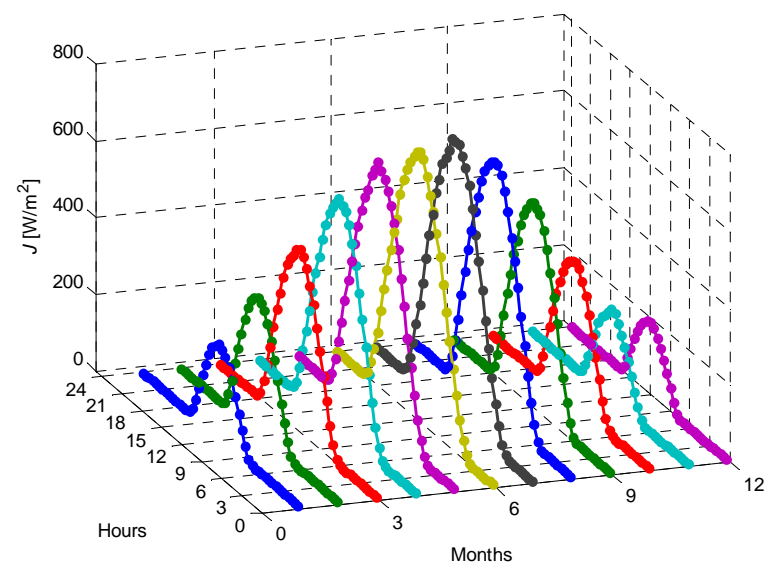

Fig. 2. Average values of measured solar irradiance

For consumption in those characteristic days the load profile shown in Fig. 3 is applied to each individual load.

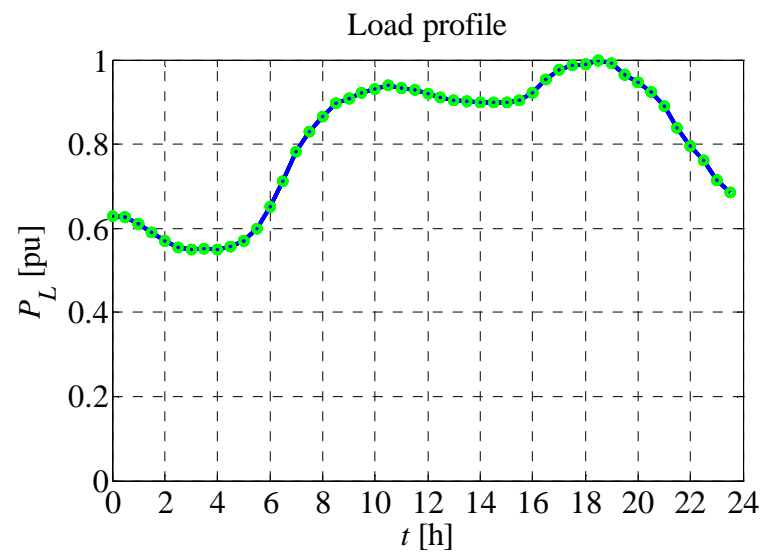

Fig. 3. Load profile used in analysis[8]

The per unit load profile is after that multiplied with the rated power of each load in order to determine its active power. The reactive power of loads is considered as inductive with the power factor of 0.9. In this way, the active and reactive power profiles are determined for all loads in the three discussed networks. In the following subsections the results for urban, rural and rural network with long distance LV lines are presented.

\section{A. Urban network}

The analysed urban network is presented in Fig. 4. It has one $47 \mathrm{kWp}$ PV system and peak load of $331 \mathrm{~kW}$. It is connected to the medium voltage network through a 1000 kVA transformer with connection type Dyn5, $10 \mathrm{kV} / 0.4 \mathrm{kV}, \quad u_{k}=5.82 \%, \quad P_{F e 0}=1.75 \mathrm{~kW} \quad$ and $P_{C u 0}=13.5 \mathrm{~kW}$.

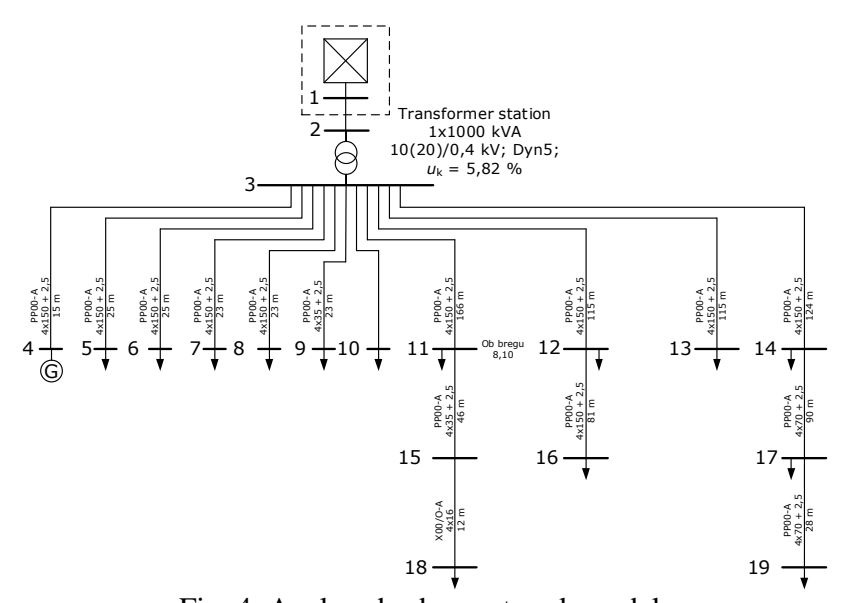

Fig. 4. Analysed urban network model

For the discussed low voltage distribution network, two analyses are performed. The first analysis deals with the energy savings achieved with existing PV system. The second analysis is performed for a hypothetical installation of smaller PV systems instead of a single 47 $\mathrm{kWp}$ PV system. In the second case the PV systems of 5 $\mathrm{kWp}$ are installed at the nodes $4,5,6,8,11,12,14,17$ and $7 \mathrm{kWp}$ at the node 13 . The results of energy savings due to reactive power generation in PV systems are presented in Table I.

Table I. Results of yearly energy savings for presented urban network

\begin{tabular}{|c|c|c|c|}
\cline { 2 - 4 } \multicolumn{1}{c|}{} & $\begin{array}{c}W_{\text {loss }} \\
{[\mathrm{kWh}]}\end{array}$ & $\begin{array}{c}W_{\text {loss }, L V} \\
{[\mathrm{kWh}]}\end{array}$ & $\begin{array}{c}W_{\text {loss } T} \\
{[\mathrm{kWh}]}\end{array}$ \\
\hline $\begin{array}{c}\text { Existing PV } \\
\text { systems }\end{array}$ & 160.1 & 6.4 & 153.7 \\
\hline $\begin{array}{c}\text { Smaller PV } \\
\text { systems }\end{array}$ & 425.7 & 168.7 & 156.0 \\
\hline
\end{tabular}

Since the observed losses consist of two parts, the energy savings are also presented in this way. $W_{\text {loss }, L V}$ are the energy savings achieved by reducing energy losses in the low voltage network, whilst $W_{\text {loss }, T}$ are the energy savings achieved by the reduced losses in distribution transformer. From the results shown in Table I following conclusions can be drown. The reactive power generation in the existing PV system can reduce losses in the transformer, but not in the LV network. The reason for that is the connection of PV system directly to the transformer busbar. Therefore the reactive power generated in PV system only reduces the reactive power delivered through the transformer, but it does not influence the losses in other part of the network. On the other hand, the results 
presented in Table I clearly show, that major decrease in energy losses could be achieved by replacing a single PV system connected to the transformer busbar by several smaller PV systems distributed in the network. In this way not only transformer but also the LV network losses can be reduced.

\section{B. Rural network}

The rural network model is presented in Fig. 5. It has two $50 \mathrm{kWp}$ PV systems connected to the nodes 5 and 23. The peak loading of the network equals $115 \mathrm{~kW}$. The connection to the $20 \mathrm{kV}$ medium voltage network is made through a $250 \mathrm{kVA}$ transformer, Dyn5, $20 \mathrm{kV} / 0.4 \mathrm{kV}$, $u_{k}=4.0 \%, P_{F e 0}=0.6 \mathrm{~kW}$ and $P_{C u 0}=3.25 \mathrm{~kW}$.

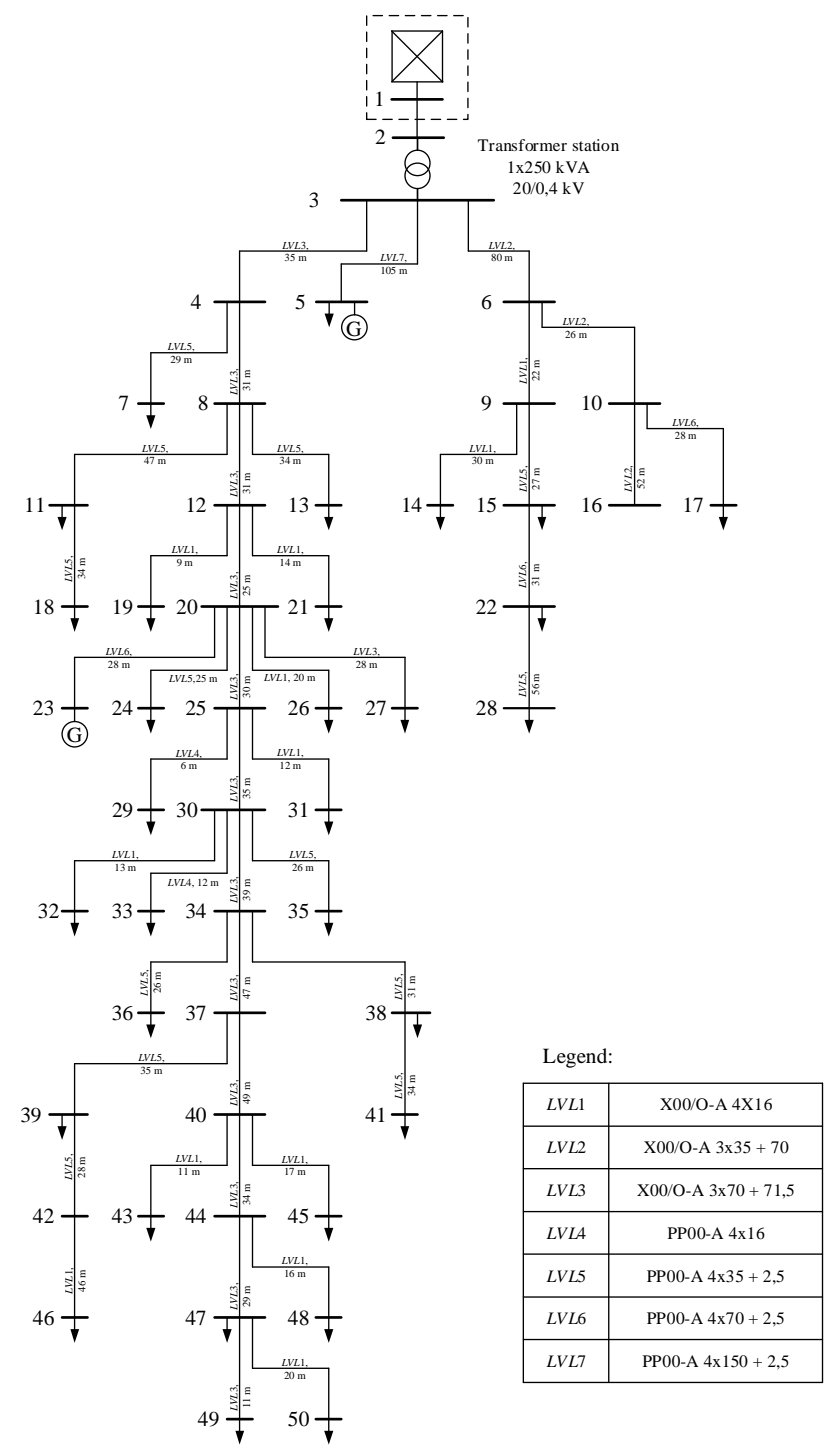

Fig. 5 Analysed rural network model

For LV network presented in Fig. 5 the same two analyses are performed as those described in the previous subsection. The first analysis deals with the yearly energy savings that can be achieved by a proper reactive power generation considering the existing installed powers and locations of PV systems. In the second analysis the existing PV systems are replaced with smaller, $5 \mathrm{kWp} \mathrm{PV}$ systems, with the same total power. These PV systems are dispersed over the network. They are installed in the nodes $5,7,11,13,14,15,18,19,21,22,23,24,26,27$, $29,31,32,33,35$, and 36 . The yearly energy savings that can be achieved by a proper reactive power generation in PV systems are presented in table II for both discussed cases.

Table II. Results of yearly energy savings for presented

\begin{tabular}{|c|c|c|c|}
\cline { 2 - 4 } \multicolumn{1}{c|}{} & $\begin{array}{c}W_{\text {loss }} \\
{[\mathrm{kWh}]}\end{array}$ & $\begin{array}{c}W_{\text {loss }, L V} \\
{[\mathrm{kWh}]}\end{array}$ & $\begin{array}{c}W_{\text {loss, }} \\
{[\mathrm{kWh}]}\end{array}$ \\
\hline $\begin{array}{c}\text { Existing PV } \\
\text { systems }\end{array}$ & 1147.6 & 850.4 & 297.2 \\
\hline $\begin{array}{c}\text { Smaller PV } \\
\text { systems }\end{array}$ & 1697.6 & 1387.7 & 309.9 \\
\hline
\end{tabular}

From the results presented in Table II following conclusions can be drown. In the case of existing PV systems energy savings equal $1147.6 \mathrm{kWh}$. The majority of these savings is achieved by the reduction of LV network losses. Reason for that is the installation of one PV system away from transformer busbar, whilst the second one is still connected there. In the case with smaller PV systems, where the majority of them is distributed and connected to the network away from the transformer busbar, higher energy savings of $1697.6 \mathrm{kWh}$ can be achieved. However, when these losses are compared with the ones achieved with the existing PV systems, it becomes evident that the energy savings in the transformer did not change substantially, since in both cases almost maximal reduction of transformer losses is achieved. Thus, for the reduction of transformer losses, the site of PV system installation is unimportant.

The comparison of results, given in Tables I and II for discussed urban and rural network, clearly shows that higher energy savings are achieved in the rural network (Table II). The rural network is weakly sized. Its lines are longer whilst the conductor cross-sections are smaller. This means that higher losses occur in normal operation of the rural network, which can be reduced in part by local reactive power generation in PV systems.

\section{Rural network with long distance LV lines}

In this subsection the same analyses as in previous two subsections are performed in the case of rural network where even longer distances of LV lines are present. The discussed network is presented in Fig. 6. It contains only one $10 \mathrm{kWp}$ PV system, connected to the node 7. The peak loading of the network equals $44.7 \mathrm{~kW}$. The connection to the medium voltage network is made through a $50 \mathrm{kVA}$ transformer $\mathrm{Yz} 5,20 \mathrm{kV} / 0.4 \mathrm{kV}$, $u_{k}=4.0 \%, P_{F e 0}=0.2 \mathrm{~kW}$ and $P_{C u 0}=1.05 \mathrm{~kW}$. In the first analysis yearly energy savings are determined for discussed network with existing PV system. In the second analysis, two smaller $5 \mathrm{kWp} \mathrm{PV} \mathrm{systems} \mathrm{are} \mathrm{installed} \mathrm{in}$ the nodes 24 and 26 instead of the original $10 \mathrm{kWp} \mathrm{PV}$ system. Considering these 2 PV systems possible energy savings are evaluated again. The results of performed analysis - energy savings gathered by reduced energy losses due to the optimal PV system reactive power generation, are presented Table III. 


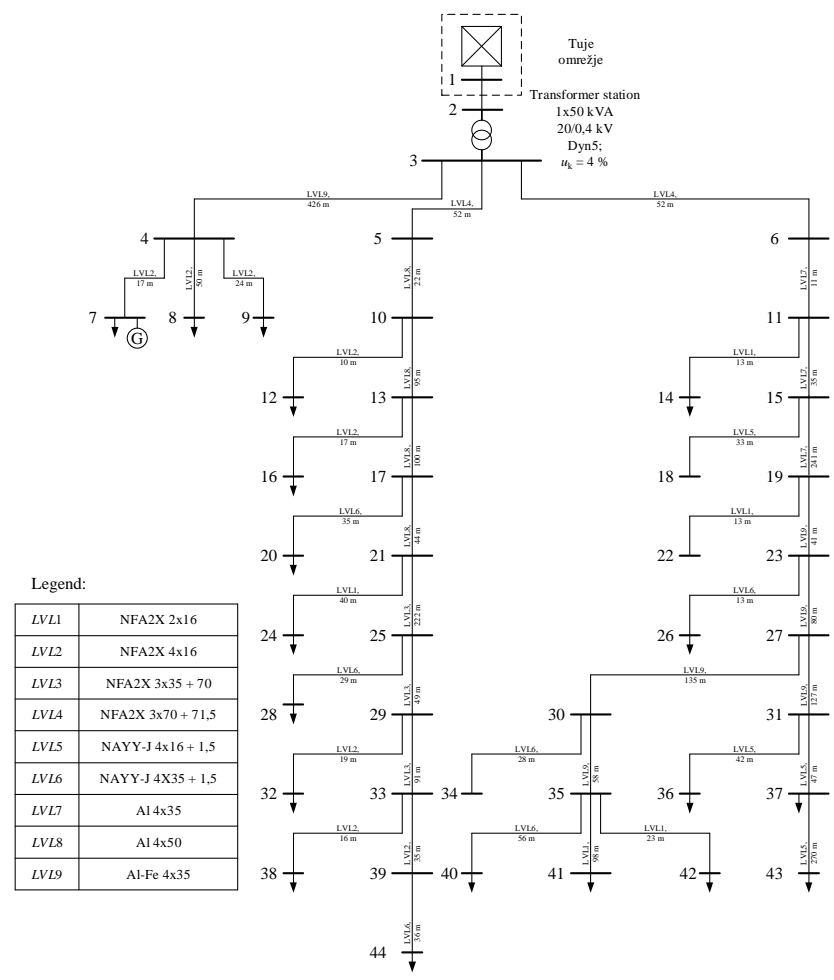

Fig. 6 Analysed rural network with long distance LV lines

Table III Results of yearly energy savings for rural network with long distance LV lines

\begin{tabular}{|c|c|c|c|}
\cline { 2 - 4 } \multicolumn{1}{c|}{} & $\begin{array}{c}W_{\text {loss }} \\
{[\mathrm{kWh}]}\end{array}$ & $\begin{array}{c}W_{\text {loss }, L V} \\
{[\mathrm{kWh}]}\end{array}$ & $\begin{array}{c}W_{\text {loss } T} \\
{[\mathrm{kWh}]}\end{array}$ \\
\hline $\begin{array}{c}\text { Existing PV } \\
\text { systems }\end{array}$ & 267.2 & 122.5 & 144.6 \\
\hline $\begin{array}{c}\text { Smaller PV } \\
\text { systems }\end{array}$ & 332.9 & 186.0 & 146.9 \\
\hline
\end{tabular}

The results presented in Table III show, that even one existing PV system with installed power of $10 \mathrm{kWp}$ can bring yearly energy savings of $267.2 \mathrm{kWh}$. These energy savings were achieved by reduction of both, transformer and LV network losses. When smaller PV systems instead of big one are installed in the network, the energy savings raises due to higher energy savings in LV network and equals $332.9 \mathrm{kWh}$.

The comparison of results, given in Tables I and III for existing PV systems clearly shows, that higher energy savings are achieved in the rural network with long distance LV lines (Table III), where nearly five times smaller PV system is installed than in the urban network (Table I).

These results show that for energy savings it is important where PV systems are installed. The installation of PV systems in the network can benefit in reducing both, the LV network and transformer losses, whilst installation to the transformer busbar can only reduce the transformer losses. According to this the PV systems should preferably be connected in the network. In this way their impact on both, the transformer and the LV network losses, can be maximized.

\section{Conclusion}

In order to improve the operation of low voltage network with installed PV systems it is desirable that PV systems generate also reactive power. However, if the objective of such reactive power generation is a reduction of energy losses, the reactive power must be generated according to the network needs. In this paper energy savings for discussed urban, rural and rural low voltage network with long distance LV lines are discussed. It is shown that energy savings due to reduced losses can be achieved with such reactive power generation. Higher energy savings can be achieved, if several smaller PV systems are installed, at the sites where consumption is present, instead of a single big PV system connected directly to the busbar of the transformer.

\section{Acknowledgement}

This work was supported in part by Slovenian Research Agency under research contract L2-5489.

\section{References}

[1] C. S. Psomopoulos, I. Skoula, C. Karras, A. Chatzimpiros, and M. Chionidis, "Electricity savings and $\mathrm{CO} 2$ emissions reduction in buildings sector: How important the network losses are in the calculation?," Energy, vol. 35, no. 1, pp. 485-490, Jan. 2010.

[2] S. Kalambe and G. Agnihotri, "Loss minimization techniques used in distribution network: bibliographical survey," Renew. Sustain. Energy Rev., vol. 29, pp. 184-200, Jan. 2014.

[3] E. Demirok, P. C. Gonzéles, K. H. B. Frederiksen, D. Sera, P. Rodriguez, and R. Teudorescu, "Local Reactive Power Control Methods for Overvoltage Prevention of Distributed Solar Inverters in Low-Voltage Grids," IEEE J. Photovoltaics, vol. 1, no. 2, pp. 174-182, 2011.

[4] D. Thukaram, H. M. Wijekoon Banda, and J. Jerome, "A robust three phase power flow algorithm for radial distribution systems," Electr. Power Syst. Res., vol. 50, no. 3, pp. 227-236, Jun. 1999.

[5] A. Vargas and M. E. Samper, "Real-time monitoring and economic dispatch of smart distribution grids: High performance algorithms for DMS applications," IEEE Trans. Smart Grid, vol. 3, no. 2, pp. 866-877, 2012.

[6] M. Braun, "Reactive power supply by distributed generators," 2008 IEEE Power Energy Soc. Gen. Meet. Convers. Deliv. Electr. Energy 21st Century, pp. 1-8, Jul. 2008.

[7] "Voltage characteristics of electricity supplied by public distribution systems," EN 50160, 2011.

[8] F. Gubina, S. Gašperič, and D. Gerbec, "Determining load profiles of Slovenian distribution network /Izdelava nadomestnih obremenitvenih diagramov za slovensko distribucijsko omrežje," University of Ljubljana, Faculty of Electrical Engineering, Research report, Ljubljana, 2004. 